\title{
Erratum to: Threshold Boolean form for joint probabilistic constraints with random technology matrix
}

\author{
Alexander Kogan' ${ }^{1}$ Miguel A. Lejeune ${ }^{2}$. \\ James Luedtke ${ }^{3}$
}

Published online: 11 December 2015

(C) Springer-Verlag Berlin Heidelberg and Mathematical Optimization Society 2015

\section{Erratum to: Math. Program. Ser. A (2014) 147:391-427 DOI 10.1007/s10107-013-0728-y}

This note reports a correction and clarifies the results presented in [2]. The third author of this corrigendum is not an author of the paper [2]. In the original publication, Theorem 28 (Sect. 4.2.1, p. 410) states that "The probabilistically constrained problem PMRTM can be reformulated as the quadratic integer optimization problem QDE." This statement must be corrected, since problem QDE is in fact an inner approximation of the chance-constrained problem PMRTM, and the equivalence between QDE and PMRTM can only be claimed under the specific settings presented below in the modified Theorem 28.

In part (ii) of Theorem 28 in [2, p. 411], it is shown that any feasible solution for the chance-constrained problem PMRTM defined by a $p$-sufficient recombination $\omega^{k}$ is also feasible for the reformulated problem QDE. While that statement is correct, this, along with the fact any feasible solution of QDE is feasible for PMRTM (part

The online version of the original article can be found under doi:10.1007/s10107-013-0728-y.

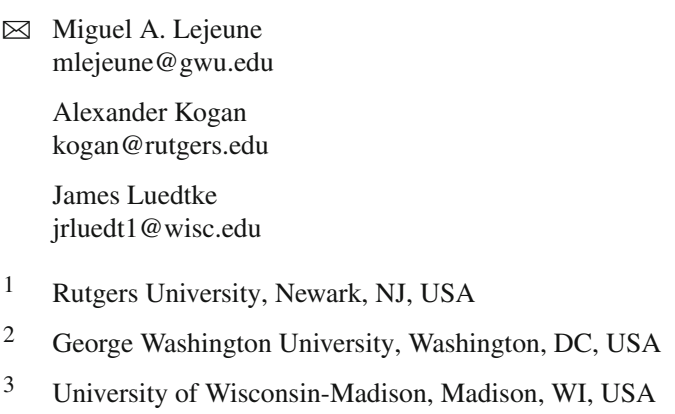


Table 1 Possible realizations of $\xi$ in Example 1

\begin{tabular}{lllllll}
\hline$k$ & $\omega_{1}^{k}$ & $\omega_{2}^{k}$ & $\mathbb{P}\left(\xi=\omega^{k}\right)$ & $F_{1}\left(\omega_{1}^{k}\right)$ & $F_{2}\left(\omega_{2}^{k}\right)$ & $F\left(\omega^{k}\right)$ \\
\hline 1 & 1 & 2 & $1 / 3$ & $1 / 3$ & 1 & $1 / 3$ \\
2 & 2 & 1 & $1 / 3$ & 1 & $1 / 3$ & $1 / 3$ \\
3 & 2 & 2 & $1 / 3$ & 1 & 1 & 1 \\
\hline
\end{tabular}

(i) of Theorem 28 [2, p. 410]), is not sufficient to claim the equivalence between QDE and PMRTM. Indeed, it might be possible to find feasible solutions for the chanceconstrained problem PMRTM that do not correspond to a $p$-sufficient recombination $k \in \bar{\Omega}_{B}^{+}$and that are not be feasible for QDE.

The following example [5] illustrates that the reformulation QDE proposed in [2] is not in general equivalent to PMRTM, but constitutes an inner approximation of the chance-constrained problem PMRTM.

Example 1 Let $d=3$, and let the three possible realizations of $\xi$ be given in Table 1, along with the joint cumulative probability function $F$ and the marginal cumulative distribution functions $F_{j}, j=1,2$.

Set $p=2 / 3$ so that in order to be feasible to the probabilistic constraint a solution $x$ must satisfy at least two of the following three inequalities:

$$
x_{1}+2 x_{2} \leq 3, \quad 2 x_{1}+x_{2} \leq 3, \quad 2 x_{1}+2 x_{2} \leq 3
$$

Because the last inequality dominates the other two, the feasible region reduces to:

$$
G=\left\{x \in \mathbb{R}_{+}^{2}: x_{1}+2 x_{2} \leq 3,2 x_{1}+x_{2} \leq 3\right\} \text {. }
$$

We next present the QDE formulation for this example. The sets of cut points are: $C_{1}=\left\{\omega_{j}^{k}: F_{j}\left(\omega_{j}^{k}\right) \geq p, k \in \Omega\right\}=\{2\}$ and $C_{2}=\{2\}$. Thus, the only recombination is the vector $(2,2)$, which satisfies $F((2,2))=1 \geq p$, and the set $\bar{\Omega}_{B}^{-}$is empty. Using the equation numbering in [2], we see that: (1) there is no constraint (33); (2) the constraints (34) are $\lambda_{11}=1$ and $\lambda_{21}=1$; and 3) the constraint (62) is:

$$
x_{1}\left(2 \lambda_{11}\right)+x_{2}\left(2 \lambda_{21}\right) \leq 3
$$

Substituting in the only feasible $\lambda$ solution we obtain:

$$
X=\left\{x \in \mathbb{R}_{+}^{2}: 2 x_{1}+2 x_{2} \leq 3\right\} .
$$

Thus, $(1,1) \in G$ but $(1,1) \notin X$.

\section{Revised statement of Theorem 28 in [2]}

We provide now the revised Theorem 28 which defines several conditions under which the formulation QDE is an exact reformulation for PMRTM. 
Using $t_{i j}=s_{i j} \xi_{j}$ in [2], the joint chance constraint (3) in [2, p. 392] can be rewritten as

$$
\mathbb{P}\left(\sum_{j \in J} t_{i j} x_{j} \leq d_{i}, i=1, \ldots, r\right) \geq p
$$

The modified Theorem 28 is given below.

Theorem 28 The quadratic integer optimization problem QDE is in general an inner approximation of the probabilistically constrained problem PMRTM, and is equivalent to the probabilistically constrained problem PMRTM if any of the following conditions are satisfied:

(i) Each inequality in (1) has exactly one monomial:

$$
\mathbb{P}\left(x_{j} t_{i j} \leq d_{i}, i=1, \ldots, r\right) \geq p
$$

(ii) Each inequality in (1) contains exactly one random variable:

$$
\mathbb{P}\left(t_{i} \sum_{j=1}^{m} x_{j} \leq d_{i}, i=1, \ldots, r\right) \geq p,
$$

with $t_{i}=t_{i j}, j \in J$ for each $i$.

(iii) Each inequality in (1) contains exactly one decision variable:

$$
\mathbb{P}\left(x_{j^{\prime}} \sum_{j=1}^{m} t_{i j} \leq d_{i}, i=1, \ldots, r\right) \geq p,
$$

with $j^{\prime} \in J$.

(iv) Each inequality in (1) is the product of a sum of random variables by a sum of decision variables:

$$
\mathbb{P}\left(\sum_{k=1}^{m} t_{i k} \sum_{j=1}^{m} x_{j} \leq d_{i}, i=1, \ldots, r\right) \geq p
$$

(v) The system of inequalities in (1) includes any combination of stochastic inequalities of the form described in (i) to (iv).

Proof It was shown in the original manuscript [2] that: (1) any solution feasible for QDE is feasible for PMRTM, and (2) any solution feasible for PMRTM defined by $k \in \bar{\Omega}^{+}$is feasible for QDE. What remains to be demonstrated is that any solution feasible for PMRTM is always feasible for QDE when the chance constraint takes a form presented in (i) to (v). 
(i): $x_{j} t_{i j} \leq d_{i}$ can be rewritten as: (a) $\frac{x_{j}}{d_{i}} \leq \frac{1}{\xi_{i j}} \Leftrightarrow-\frac{x_{j}}{d_{i}} \geq-\frac{1}{t_{i j}}$ if $d_{i}$ and $t_{i j}$ have the same sign, and (b) $\frac{x_{j}}{d_{i}} \geq \frac{1}{t_{i j}}$ if $d_{i}$ and $t_{i j}$ have opposite signs. This corresponds to the case of a joint chance constraint with random right-hand side vector. When the uncertainty is the random right-hand side only, it is well known (see, e.g., $[1,3])$ that there is no $k^{\prime} \notin \bar{\Omega}_{B}^{+}$such that $\mathbb{P}\left(\xi \leq \omega^{k^{\prime}}\right) \geq p$ with $\omega^{k^{\prime}} \leq \omega^{k}$ and $\omega^{k^{\prime}} \neq \omega^{k}$ for any $k \in \bar{\Omega}_{B}^{+}$. Hence, under condition (i), the feasible area defined by PMRTM is included in the feasible area defined by QDE, which is the result that we set out to prove.

(ii): As in (i), we have a single random variable in each stochastic inequality.

(iii): Let $\vartheta_{i}=\sum_{j=1}^{m} t_{i j}, i=1, \ldots, r$. Constraint (4) becomes

$$
\mathbb{P}\left(\vartheta_{i} x_{i} \leq d_{i}, i=1, \ldots, r\right) \geq p
$$

As $\xi_{i j}$ is discretely distributed with finite support, so is $\vartheta_{i}$. As (6) has the same form as (2), the proof of (i) applies here too.

(iv): The probabilistic constraint (5) can be rewritten as

$$
\mathbb{P}\left(\vartheta_{i} \sum_{j=1}^{m} x_{j} \leq d_{i}, i=1, \ldots, r\right) \geq p
$$

which is similar to (3).

(v): The proofs of (i)-(iv) essentially show that each stochastic inequality is equivalent to a collection of inequalities of type (i).

The proof is obtained with the same approach used in [4] in the case of joint chance constraints including quadratic stochastic inequalities of the form $\sum_{j_{1}, j_{2} \in J} s_{i j_{1} j_{2}} x_{j_{1}} x_{j_{2}} \xi_{i j_{1} j_{2}} \leq d_{i}, i=1, \ldots, r$ instead of the linear ones considered in $[2]$.

\section{References}

1. Dentcheva, D., Prékopa, A., Ruszczyński, A.: Concavity and efficient points of discrete distributions in probabilistic programming. Math. Progr. 47(3), 1997-2009 (2001)

2. Kogan, A., Lejeune, M.A.: Threshold Boolean form for joint probabilistic constraints with random technology matrix. Math. Progr. 147, 391-427 (2014)

3. Lejeune, M.A.: Pattern-based modeling and solution of probabilistically constrained optimization problems. Oper. Res. 60(6), 1356-1372 (2012)

4. Lejeune, M.A., Margot, F.: Solving Chance-Constrained Optimization Problems with Stochastic Quadratic Inequalities. Tepper Working Paper 2014-E8, Carnegie Mellon University, PA. http://wpweb2. tepper.cmu.edu/fmargot/PDF/trilinear.pdf (2014)

5. Luedtke, J.: A Counter Example to a Threshold Boolean Form for Joint Probabilistic Constraints with Random Technology Matrix. Optimization. http://www.optimization-online.org/DB_HTML/2015/01/ 4716.html (2015) 